\title{
Effect of Albumin on the Disappearance of L-Tryptophan from the Perfusate into Isolated Perfused Rat Livers
}

\author{
Emi Sasaki, ${ }^{1}$ Yoshiji Ohta, ${ }^{1}$ Rikio Shinohara, ${ }^{2}$ \\ and Isao ISHIGURO ${ }^{1, *}$ \\ ${ }^{1}$ Department of Biochemistry, School of Medicine, and ${ }^{2}$ Department of \\ Biochemistry, School of Health Sciences, Fujita Health University, \\ Toyoake 470-11, Japan
}

(Received May 27, 1993)

Summary In order to clarify the role of albumin in the transport of L-tryptophan ( $\operatorname{Trp}$ ) into the liver, we examined the effect of bovine serum albumin (BSA) on the disappearance of Trp from the perfusate into isolated perfused rat livers. In liver perfusion with the perfusion medium containing $100 \mu \mathrm{M} \operatorname{Trp}$ and $0.5,2$, or $4 \%$ BSA, the disappearance of Trp from the perfusate was depressed with a decrease in the concentration of free Trp (albumin-unbound form). The perfusion of livers with the medium containing $1 \mathrm{~mm}$ Trp and $0.5,2$, or $4 \%$ BSA had little effect on the disappearance of Trp from the perfusate, though free-Trp concentrations decreased with perfusion time. In the perfusate of liver perfusion with the medium containing $100 \mu \mathrm{M}$ Trp and $0.5 \%$ BSA, total Trp concentrations correlated well with free Trp concentrations, whereas when the perfusion medium contained $100 \mu \mathrm{M}$ Trp and 2 or 4\% BSA, total Trp concentrations correlated well with bound Trp concentrations. When liver perfusion was conducted with the medium containing $1 \mathrm{mM}$ Trp with $0.5,2$, or $4 \%$ BSA, there was a good correlation between total Trp and free Trp concentrations in the perfusate. These results suggest that under physiological conditions (ca., $100 \mu \mathrm{M}$ total Trp and $4 \%$ albumin), albumin contributes to the maintenance of total serum Trp concentrations and to the constant supply of serum Trp to the liver by lowering changes in free serum Trp concentrations through its binding to the amino acid.

Key Words: L-tryptophan, albumin, hepatic transport, isolated perfused liver (rat)

${ }^{*}$ To whom correspondence should be addressed. 
L-Tryptophan (Trp) is one of the nutritionally essential amino acids. In the plasma or serum of humans and animals, $80-90 \%$ of the total Trp is normally present in an albumin-bound form, while the remainder (10-20 $\mu \mathrm{M})$ circulates in a free form [1-4]. More than $90 \%$ of the total Trp is known to be converted to kynurenine and subsequently to niacin and NAD in the liver through the kynurenine pathway in which tryptophan 2,3-dioxygenase takes part under physiological conditions [5]. The remaining Trp is available for not only protein synthesis in various tissues but also the synthesis of 5-hydroxytryptamine (serotonin), a neurotransmitter in the brain, through the 5-hydroxyindole pathway in which tryptophan hydroxylase takes part [5]. Recently, it was demonstrated that in addition to serotonin, quinolinic acid, which is synthesized in the brain through the kynurenine pathway in which indoleamine 2,3-dioxygenase participates, is an important member of the family of neuroactive compounds [6]. Trp itself was shown to stimulate liver protein synthesis in rats and mice [7]. Thus, Trp is a nutritionally and physiologically important amino acid.

There are some reports on the transport of Trp into isolated hepatocytes [4, $8-10]$ and isolated perfused livers $[11,12]$, but the effect of albumin on the hepatic transport of the amino acid was not studied in these experiments. Fatty acids and drugs such as salicylate and diazepam, which bind to albumin more strongly than Trp, are known to affect the binding of Trp to albumin [13, 14]. Smith and Pogson [15] reported that the rate of Trp catabolism in isolated rat hepatocytes is enhanced by increasing free Trp concentrations in the medium containing Trp and bovine serum albumin (BSA) by addition of palmitic acid to the medium, and concluded that alterations in free Trp concentrations consequent to changes in albumin binding may be an important factor in the regulation of Trp uptake and catabolism by the liver. In addition, Salter et al. [16] showed that a rapid dissociation of Trp from albumin and a large fractional extraction of the free pool of Trp during passage through the liver are necessary factors to consider in determining the effect of the bound pool of Trp on liver Trp catabolism. From these findings, it can be presumed that free Trp is taken up into liver cells more easily than bound Trp and that albumin should regulate hepatic Trp uptake through the control of free Trp concentrations in the blood by binding to the amino acid. However, the exact role of albumin in the transport of Trp into the liver is unclear at present.

Therefore, in the present study we examined the effect of albumin on the disappearance of Trp from the perfusate containing various concentrations of Trp with and without BSA during the perfusion of isolated rat livers, because experiments using isolated perfused livers can be carried out not only under very nearly in vivo conditions but also under the direct observation of metabolic changes in the liver alone. 


\section{MATERIALS AND METHODS}

Experimental animal. Male Wistar rats weighing 250-300 g were employed in the present experiments and were fed chow (Oriental MF, Oriental Yeast Co., Tokyo) ad libitum for at least 1 week before use.

Chemicals. Trp (the highest purity grade) was purchased from Wako Pure Chemical Ind., Co. (Osaka). BSA (fraction V) was obtained from Sigma Chemical Co. (St. Louis, MO). All other chemicals used were of the highest purity grade and were also obtained commercially.

Liver perfusion. Liver perfusion was carried out at $37^{\circ} \mathrm{C}$ over a period of 120 min by the method of Miller et al. [17]. The perfusion medium consisted of Henseleit bicarbonate buffer $(\mathrm{pH} 7.4)$ containing $10 \mathrm{~mm}$ glucose and various concentrations of Trp with and without BSA. The rate of perfusion was $2 \mathrm{ml} / \mathrm{min}$ per $\mathrm{g}$ liver. The volume of the perfusion medium used for this liver perfusion was $120 \mathrm{ml}$, and sampling of the perfusate $(2 \mathrm{ml})$ was performed every $15 \mathrm{~min}$ during the first $60 \mathrm{~min}$ of perfusion and every $30 \mathrm{~min}$ thereafter.

Trp determination. Trp in the perfusate was measured by high-performance liquid chromatography (HPLC) with electrochemical detection as described previously [4]. A Yanagimoto L-5000 pump (Yanaco Co., Kyoto), a Yanapak ODS-T (4 $\phi-250 \mathrm{~mm}$, Yanaco Co.), and a volutametry detector VMO-101A (Yanaco Co.) were used in this HPLC system. The chromatography was conducted at $40^{\circ} \mathrm{C}$ with a solution of $0.1 \mathrm{M}$ citric acid- $0.1 \mathrm{M}$ sodium acetate and methanol (4: $1 \mathrm{v} / \mathrm{v}$ ) used as an elution solution, and the flow rate was $1.0 \mathrm{ml} / \mathrm{min}$. After the perfusate sample had been mixed with $0.4 \mathrm{M}$ perchloric acid, the mixture was centrifuged at $15,000 \times g$ for $10 \mathrm{~min}$. The resulting supernatant was used for determination of total Trp. Another aliquot of perfusate was centrifuged at $1,500 \times$ $g$ for $30 \mathrm{~min}$ in a Centriflo membrane cone CF 25 (Amicon Grace Co., Tokyo), and the filtrate was used for determination of free Trp. The concentration of albumin-bound Trp was estimated from the difference between total Trp and free Trp concentrations determined. The ratio of binding of Trp to albumin (bound Trp/total Trp) was expressed as a percentage.

Kinetic analysis. The rate constant of Trp disappearance from the perfusate and its half life were analyzed by the one-compartment model [18] and calculated by use of an iterative least-squares computer program, MULTI [19], adapted for use by a personal computer FM-16 $\beta$ (Fujitsu, Tokyo).

Statistics. All values obtained were expressed as means \pm SD. If necessary, results were analyzed statistically by Student's $t$-test. The level of significance was set at $p<0.05$.

\section{RESULTS}

We first examined the effect of BSA on Trp disappearance from the perfusate Vol. 15, No. 3, 1993 
following liver perfusion using $100 \mu \mathrm{M}$ Trp. BSA was added to this perfusion medium in a final concentration of $0.5,2$, or $4 \%$. The free-Trp concentration in the perfusion medium containing $100 \mu \mathrm{M}$ Trp with 0.5 , 2, or $4 \%$ BSA was $73.1 \pm 0.9$, $24.9 \pm 9.2$, or $4.4 \pm 2.1 \mu \mathrm{M}$, respectively. When liver perfusion was conducted with the perfusion medium containing $100 \mu \mathrm{M}$ Trp without BSA over a period of 120 min, Trp concentration in the perfusate decreased rapidly with perfusion time during the first $60 \mathrm{~min}$, at which time the Trp concentration was $28.3 \pm 6.3 \mu \mathrm{M}$ (Fig. 1A). Only a slight decrease in Trp concentration was found in the perfusate thereafter (Fig. 1A). As shown in Fig. 1A, the addition of BSA to this perfusion medium had a depressive effect on the disappearance of total Trp from the perfusate; and this depressive effect was strengthened as the concentration of BSA added was increased. No significant difference in total Trp concentration between the control perfusion without BSA and the perfusion with $0.5 \%$ BSA addition was seen during liver perfusion for $120 \mathrm{~min}$, whereas a significant difference in total Trp concentration between the control perfusion and the perfusion with 2 or $4 \%$ BSA addition was observed $30 \mathrm{~min}$ after the onset of liver perfusion and thereafter (Fig. 1A). In addition, total Trp concentration in the perfusion with $0.5 \%$ BSA addition was significantly higher than that with 2 or $4 \%$ BSA addition at $60 \mathrm{~min}$ $(p<0.02)$ or $30 \mathrm{~min}(p<0.001)$, respectively, after the onset of liver perfusion (Fig. 1A).

Figure 1B, C, and D shows changes in total Trp, free Trp, and bound Trp concentrations in the perfusate following liver perfusion with the perfusion medium containing $100 \mu \mathrm{M}$ Trp with $0.5,2$, or $4 \%$ BSA, respectively. In the perfusion with $0.5 \%$ BSA addition, the change in free Trp concentration was similar to that in total Trp concentration (Fig. 1B). In the perfusion with 2 or $4 \%$ BSA addition, free Trp concentrations decreased for the first $30 \mathrm{~min}$ of liver perfusion, but no further decrease in the concentration occurred thereafter (Fig. 1C and D). In these perfusion groups, the change in total Trp concentration was similar to that in bound Trp concentration (Fig. 1C and D).

Next, liver perfusion was carried out with perfusion medium containing $1 \mathrm{~mm}$ Trp with and without BSA. The concentration of free Trp in the perfusion medium containing $1 \mathrm{~mm}$ Trp with $0.5,2$, or $4 \%$ BSA was $837 \pm 37,572 \pm 27$, or $564 \pm 41 \mu \mathrm{M}$, respectively; and so there was little difference in this concentration between 2 and 4\% BSA addition groups. In this liver perfusion without BSA, the total Trp concentration in the perfusate decreased almost linearly with perfusion time; Trp concentrations at 60 and $120 \mathrm{~min}$ after the onset of liver perfusion were $787 \pm 48$ and $565 \pm 80 \mu \mathrm{M}$, respectively (Fig. $2 \mathrm{~A}$ ). When liver perfusion was performed using the perfusion medium containing $1 \mathrm{mM}$ Trp with $0.5,2$, or $4 \%$ BSA, there was no significant difference in the disappearance of total Trp from the perfusate between the control perfusion without BSA and the perfusion with $0.5,2$, or $4 \%$ BSA addition (Fig. 2A). Under the same perfusion conditions, free Trp and bound Trp concentrations in the perfusate changed as shown in Fig. 2B, C, and D; there was a slight, but not significant, change in bound Trp concentrations, while a similar 

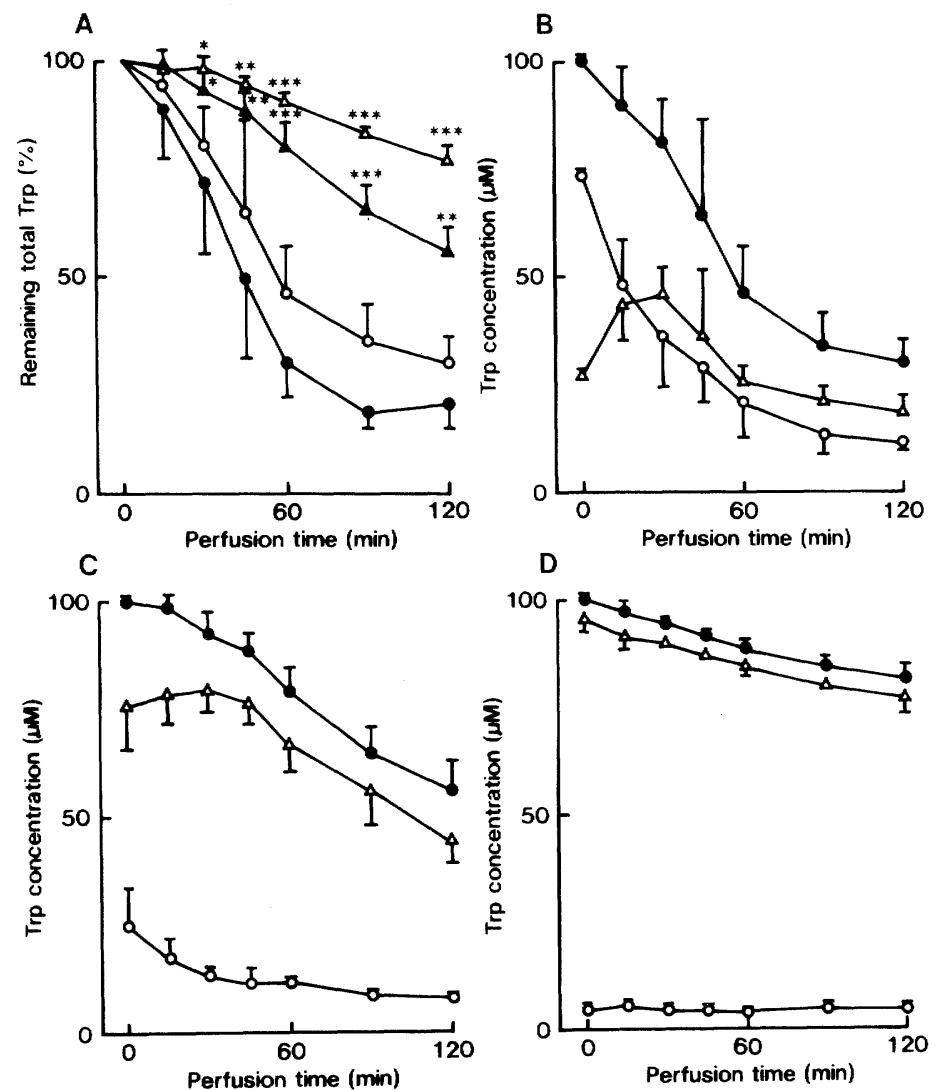

Fig. 1. Effect of various concentrations of BSA on Trp disappearance from the perfusate as a function of perfusion time during liver perfusion using $100 \mu \mathrm{M}$ Trp-containing perfusion medium. Liver perfusion and Trp determination were carried out as described in Materials and Methods. A: The percentages of total Trp remaining in the perfusate were determined for liver perfusion using perfusion medium containing $0(\bullet), 0.5(0)$, $2(\Delta)$, or $4 \%(\triangle)$ BSA. B, C, and D: Concentrations of total $(\bullet)$, free $(O)$, and bound $(\triangle)$ Trp in the perfusate were determined for liver perfusion using medium containing 0.5 (B), 2 (C), or $4 \%$ (D) BSA. Each value represents the mean $\pm \mathrm{SD}(n=3) .{ }^{*} p<0.02$, ${ }^{* *} p<0.01,{ }^{* * *} p<0.001$ vs. $0 \%$ BSA.

change was observed in total Trp and free Trp concentrations.

The ratio of binding of Trp to BSA (bound Trp/total Trp) in the perfusate during liver perfusion was next determined. The results are shown in Fig. 3. When 0.5 or $2 \%$ BSA was present in the medium containing $100 \mu \mathrm{M}$ Trp, the binding ratio increased during the first $30 \mathrm{~min}$ period of perfusion, but a further increase in the ratio did not occur thereafter. In contrast, the addition of $4 \%$ BSA to the medium containing $100 \mu \mathrm{M} \operatorname{Trp}$ and that of $0.5,2$, or $4 \%$ BSA to the medium containing $1 \mathrm{~mm}$ Trp caused little increase in the binding ratio during liver perfusion. 

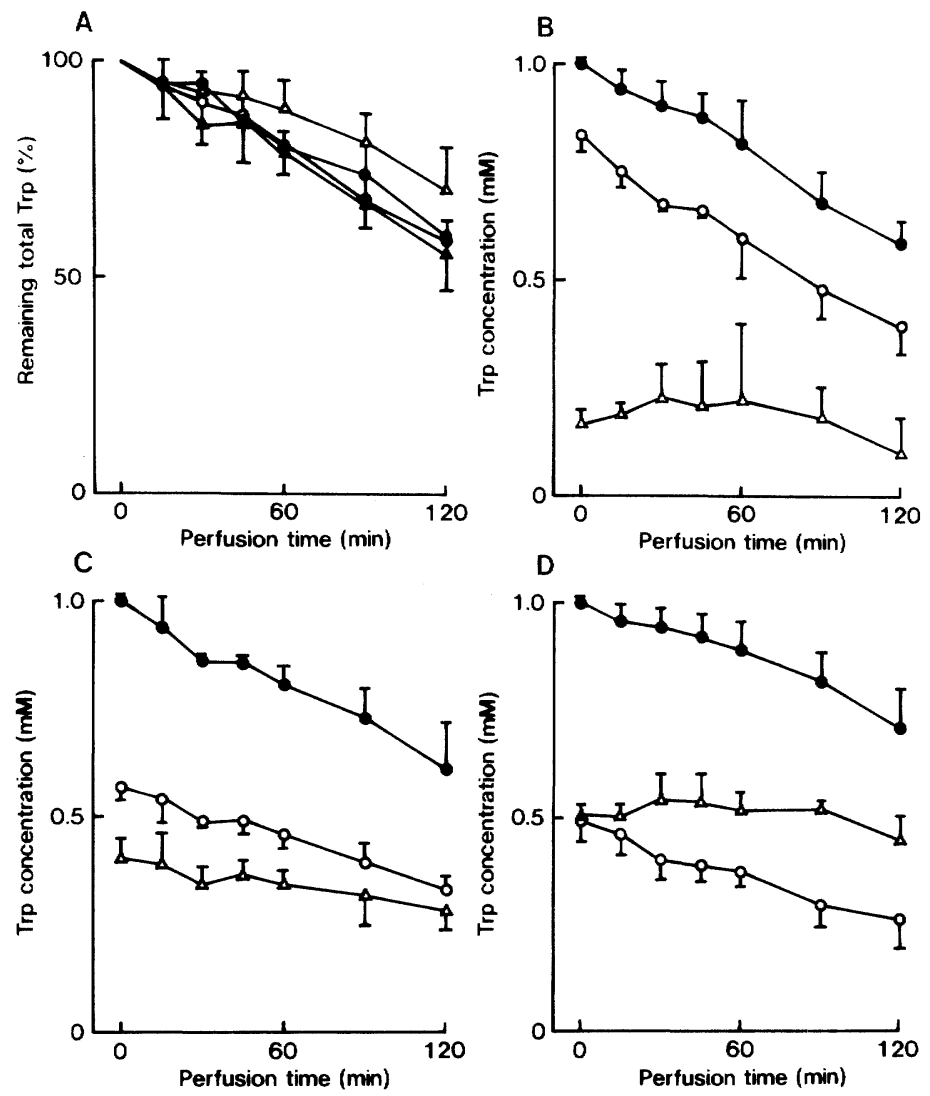

Fig. 2. Effect of various concentrations of BSA on Trp disappearance from the perfusate as a function of perfusion time during liver perfusion using $1 \mathrm{~mm}$ Trp-containing perfusion medium. Liver perfusion and Trp determination were carried out as described in Materials and Methods. A: The percentages of total Trp remaining in the perfusate were determined for liver perfusion using the perfusion medium containing $0(\bullet), 0.5$ $(\bigcirc), 2(\Delta)$, or $4 \%(\triangle)$ BSA. B, C, and D: Concentrations of total $(\bullet)$, free $(\bigcirc)$, and bound $(\triangle)$ Trp in the perfusate were determined for liver perfusion using the perfusion medium containing 0.5 (B), 2 (C), or $4 \%$ (D) BSA. Each value represents the mean \pm $\mathrm{SD}(n=3-4)$.

Since the addition of BSA to the perfusion medium containing $100 \mu \mathrm{M} \operatorname{Trp}$ caused an apparent delayed disappearance of Trp from the perfusate during liver perfusion, the rate constant of Trp disappearance and its half life were estimated from the disappearance curves during the first $60 \mathrm{~min}$ period of perfusion by the non-linear iterative least squares method. In this analysis, this liver perfusion condition was taken as a one-compartment model. The results are shown in Table 1. The disappearance rate constant $\left(\mathrm{k}_{\mathrm{d}}\right)$ obtained from the curve in the presence of 2 or $4 \%$ BSA was significantly smaller than that from the curve in the absence of BSA. In addition, there was a significant difference in the $\mathrm{k}_{\mathrm{d}}$ value between the 

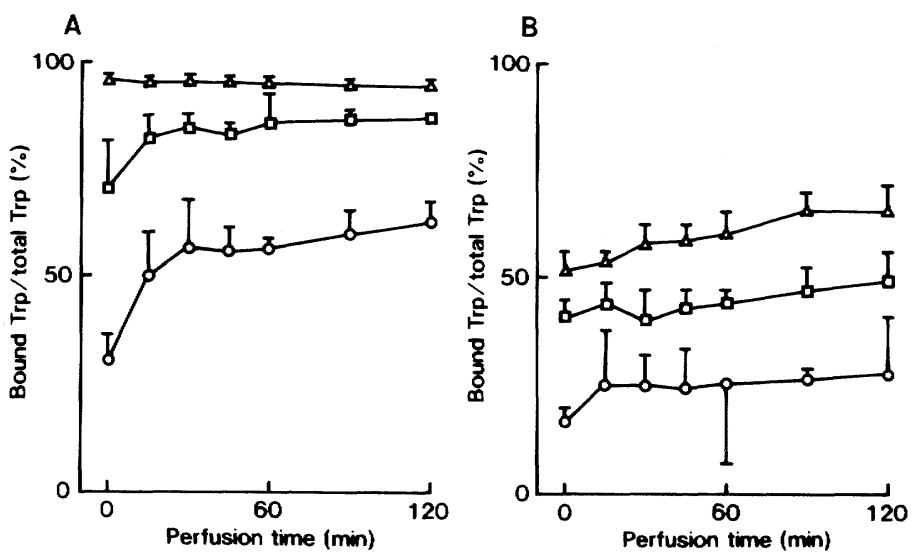

Fig. 3. Change in the ratio of binding of Trp to various concentrations of BSA in the perfusate during liver perfusion. Liver perfusion and Trp determination were carried out as described in Materials and Methods. The percentages of bound Trp/total Trp in the perfusate were determined for liver perfusion using the perfusion medium containing either $100 \mu \mathrm{M}(\mathrm{A})$ or $1 \mathrm{~mm}(\mathrm{~B})$ Trp with $0.5(\bigcirc), 2(\square)$, or $4 \%(\triangle)$ BSA. Each value represents the mean $\pm \mathrm{SD}(n=3-4)$.

Table 1. Kinetic parameters for Trp disappearance during the first $60 \mathrm{~min}$ period of liver perfusion using the perfusion medium containing $100 \mu \mathrm{M} \operatorname{Trp}$ with and without BSA.

\begin{tabular}{ccc}
\hline BSA (\%) & \multicolumn{1}{c}{$\mathrm{k}_{\mathrm{d}}\left(\mathrm{min}^{-1}\right)$} & $t_{1 / 2}(\min )$ \\
\hline 0 & $0.00229 \pm 0.00055$ & $34.3 \pm 12.6$ \\
0.5 & $0.00142 \pm 0.00044$ & $52.4 \pm 18.4$ \\
2 & $0.00058 \pm 0.00022^{* *}$ & $131.0 \pm 51.6^{*}$ \\
4 & $0.00035 \pm 0.00009^{* *}$ & $204.6 \pm 46.2^{* *}$ \\
\hline
\end{tabular}

Kinetic parameters of Trp disappearance during liver perfusion were determined from the results of three to four experiments as described in Materials and Methods. $\mathrm{k}_{\mathrm{d}}$, disappearance rate constant. ${ }^{*} p<0.05,{ }^{* *} p<0.01$ vs. $0 \%$ BSA.

Table 2. Correlation between total Trp and either free Trp or bound Trp concentrations in the perfusate during liver perfusion using perfusion medium containing either $100 \mu \mathrm{M}$ or $1 \mathrm{~mm}$ Trp with BSA.

\begin{tabular}{cccc}
\hline \multirow{2}{*}{ Trp } & \multirow{2}{*}{ BSA (\%) } & \multicolumn{2}{c}{ Correlation coefficient } \\
\cline { 3 - 4 } & & Total Trp vs. free Trp & Total Trp vs. bound Trp \\
\hline $100 \mu \mathrm{M}$ & 0.5 & $0.963^{* * *}$ & $0.840^{* * *}$ \\
& 2 & $0.692^{* * *}$ & $0.976^{* * *}$ \\
\multirow{2}{*}{$1 \mathrm{mM}$} & 0.5 & $0.677^{* *}$ & $0.995^{* *}$ \\
& 2 & $0.803^{* * *}$ & $0.463^{*}$ \\
& 4 & $0.920^{* * *}$ & $0.726^{* * *}$ \\
& & $0.800^{* * *}$ & $0.695^{* * *}$ \\
\hline
\end{tabular}

$p$ values: ${ }^{*} p<0.05,{ }^{* *} p<0.01,{ }^{* * *} p<0.001$.

presence of $0.5 \%$ BSA and either 2 or $4 \%$ BSA. The half life in the presence of 2 or $4 \%$ BSA was significantly longer than that in the absence of BSA. Furthermore, 
the half life in the presence of $4 \%$ BSA was significantly $(p<0.01)$ longer than that in the presence of $0.5 \%$ BSA.

We next examined the correlation between total Trp and either free Trp or bound Trp concentrations in the perfusate during liver perfusion using the perfusion medium containing either $100 \mu \mathrm{M}$ or $1 \mathrm{~mm}$ Trp with $0.5,2$, or $4 \%$ BSA. The results are shown in Table 2 . When liver perfusion with the medium containing $100 \mu \mathrm{M}$ Trp was conducted in the presence of 0.5 , 2, or $4 \%$ BSA, total Trp concentrations correlated well not only with free Trp concentrations but also with bound Trp concentrations. Only when $0.5 \%$ BSA was present in the medium, was the correlation between total Trp and free Trp concentrations better than that between total Trp and bound Trp concentrations. When liver perfusion with the medium containing $1 \mathrm{mM}$ Trp was conducted in the presence of $0.5,2$, or $4 \%$ BSA, the correlation between total Trp and free Trp concentrations was better than that between total Trp and bound Trp concentrations in all cases.

\section{DISCUSSION}

Although most of Trp in the plasma or serum is known to exist in an albumin-bound form [1-4] and to be metabolized in the liver [5], there has been little information on the role of albumin in the transport of the amino acid into the liver.

Therefore, using isolated perfused rat livers, we attempted to elucidate the role of albumin in liver Trp transport by examining the effect of albumin on the disappearance of Trp into the liver. When 2 or 4\% BSA was added to the perfusion medium containing $100 \mu \mathrm{M} \operatorname{Trp}$, the concentration of free $\operatorname{Trp}$ (below ca. $25 \mu \mathrm{M}$ ) was almost equal to that observed under physiological conditions. The addition of 2 or $4 \%$ BSA to the perfusion medium containing $100 \mu \mathrm{M}$ Trp was found to depress the rate of total Trp disappearance from the perfusate and to prolong its half life (Fig. 1 and Table 1). In contrast, both the addition of $0.5 \%$ BSA to the medium containing $100 \mu \mathrm{M}$ Trp and that of $0.5,2$, or $4 \%$ BSA to the medium containing 1 mM Trp, which resulted in free Trp concentrations much higher than observed under physiological conditions, were found to have little effect on the disappearance of total Trp, though free Trp concentrations decreased during liver perfusion (Figs. 1 and 2). These results suggest that under nearly physiological conditions, alterations in free Trp concentrations consequent to changes in albumin binding could affect the disappearance of total Trp from the perfusate into perfused livers.

We further determined the ratio of binding of Trp to BSA and the correlation between total Trp and either free Trp or bound Trp concentrations in the perfusate during liver perfusion in the presence of BSA. When 0.5 or $2 \%$ BSA was added to the perfusion medium containing $100 \mu \mathrm{M}$ Trp, the ratio of binding of Trp to BSA increased during the first $30 \mathrm{~min}$ period of liver perfusion and remained constant thereafter (Fig. 3). This change of the binding ratio found during the early period of liver perfusion seems to be due to a rapid uptake of Trp by the liver, resulting 
in a decrease in concentrations of free Trp which can be taken up by the liver. The addition of $4 \%$ BSA to the perfusion medium containing $100 \mu \mathrm{M}$ Trp and that of $0.5,2$, or $4 \%$ BSA to the medium containing $1 \mathrm{mM}$ Trp kept the binding ratio almost constant during liver perfusion for $120 \mathrm{~min}$, but the binding ratio with the former addition was much higher than that with the latter addition (Fig. 3). The finding that the addition of $4 \%$ BSA to the medium containing $100 \mu \mathrm{M}$ Trp causes a constant, high binding ratio between the protein and Trp suggests that under physiological conditions, albumin can keep serum Trp concentrations high and can supply it to the liver in a constant ratio. In the perfusion of livers with the perfusion medium containing $100 \mu \mathrm{M}$ Trp with 2 or $4 \%$ BSA, there was a high correlation between total Trp and bound Trp concentrations in the perfusate (Table 2). This high correlation between total Trp and bound Trp concentrations seems to be dependent on a very low concentration of free Trp because the addition of 2 or $4 \%$ BSA to the medium containing $100 \mu \mathrm{M}$ Trp led to a high binding ratio between BSA and Trp, resulting in a marked decrease in free Trp concentrations. In this condition, free Trp produced through liberation of bound Trp with a decrease in total Trp concentrations is thought to be taken up into the liver. When livers were perfused with perfusion medium containing $100 \mu \mathrm{M}$ Trp with $0.5 \%$ BSA or with medium containing $1 \mathrm{~mm} \operatorname{Trp}$ with $0.5,2$, or $4 \% \mathrm{BSA}$, total Trp concentrations in the perfusate correlated with free Trp concentrations better than with bound Trp concentrations in the perfusate (Table 2). This good correlation between total Trp and free Trp concentrations in the perfusate seems to be due to a low binding ratio between Trp and BSA, resulting in a high concentration of free Trp, which is easily taken up by the liver. These findings suggest that under physiological conditions (ca. $100 \mu \mathrm{M}$ total Trp and $4 \%$ albumin), the binding of albumin to Trp functions in the regulation of hepatic Trp transport through the level of free serum Trp concentrations and in the maintenance of total serum Trp concentration.

With respect to Trp uptake into liver cells, we demonstrated earlier that in isolated rat hepatocytes, Trp is taken up via at least two saturable transport sites (one high-affinity and one low-affinity) and one non-saturable transport one and that the high-affinity Trp transport site possesses the $K_{\mathrm{m}}$ value of $2.4 \mu \mathrm{M}$ [4]. Accordingly, under low free Trp concentrations in the perfusate, which are almost equal to those found under physiological conditions, Trp can be considered to be taken up into perfused livers via the high-affinity transport site.

In conclusion, the present results suggest that under physiological conditions, albumin contributes to the maintenance of total serum Trp concentrations and to the constant supply of serum Trp to the liver by lowering changes in free serum Trp concentrations through its binding to the amino acid.

\section{REFERENCES}

1. McMenamy, R.H., Lund, C.C., and Oncley, J.L. (1957): Unbound amino acid concentration Vol. 15, No. 3, 1993 
in human blood plasmas. J. Clin. Invest., 36, 1672-1679.

2. McMenamy, R.H., and Oncley, J.L. (1958): The specific binding of L-tryptophan to serum albumin. J. Biol. Chem., 233, 1436-1447.

3. Fuller, R.W., and Roush, B.W. (1973): Binding of tryptophan to plasma proteins in several species. Comp. Biochem. Physiol., 46B, 273-276.

4. Saito, K., Sasaki, E., Ohta, Y., Nagamura, Y., and Ishiguro, I. (1986): Mode of L-tryptophan uptake into rat hepatocytes via trypsin-sensitive high-affinity transport system. Biochem. Int., 13, 873-883.

5. Bender, D.A. (1982): Biochemistry of tryptophan in health and disease. Mol. Aspects Med., 6, 101-197.

6. Freese, A., Swartz, K.J., During, M.J., and Martin, J.B. (1990): Kynurenine metabolites of tryptophan: Implications for neurologic diseases. Neurology, 40, 691-695.

7. Sidransky, H. (1985): Tryptophan. Unique action by an essential amino acid, in Nutritional Pathology, Pathobiochemistry of Dietary Imbalances, ed. by Sidransky, H., Marcel Dekker, Inc., New York, pp. 1-62.

8. Weissbach, L., Handlogten, M.E., Christensen, H.V., and Kilberg, M.S. (1982): Evidence of two $\mathrm{Na}^{+}$-independent neutral amino acid transport systems in primary cultures of rat hepatocytes. J. Biol. Chem., 257, 12006-12011.

9. Kilberg, M.S., Weissbach, L., and Barber, E.F. (1983): Changes in amino acid transport in primary cultures of adult rat hepatocytes, in Isolation, Characterization, and Use of Hepatocytes, ed. by Harris, R.A. and Cornell, N.W., Elsevier Biomedical, New York, pp. 227-233.

10. Salter, M., Knowles, R.G., and Pogson, C.I. (1986): Transport of the aromatic amino acids into isolated rat liver cells. Biochem. J., 233, 499-506.

11. Kim, J.H., and Miller, L.L. (1969): The functional significance of changes in activity of the enzymes, tryptophan pyrrolase and tyrosine transaminase, after induction in intact rats and in the isolated, perfused rat liver. J. Biol. Chem., 244, 1410-1416.

12. Ng, C.Y., Hagino, Y., Swan, P.B., and Hederson, L.M. (1969): Metabolism of tryptophan in isolated rat livers. $J$. Nutr., 99, 465-473.

13. Curzon, G., Friedel, J., and Knott, P.J. (1973): The effect of fatty acids on the binding of tryptophan to plasma protein. Nature, 242, 198-200.

14. Hansen, U.K. (1983): Relations between high-affinity binding sites for tryptophan, diazepam, salicylate and phenol red on human serum albumin. Biochem. J., 209, 135-142.

15. Smith, S.A., and Pogson, C.I. (1980): The metabolism of L-tryptophan by isolated rat liver cells. Biochem. J., 186, 977-989.

16. Salter, M., Knowles, R.C., and Pogson, C.I. (1980): How does displacement of albuminbound tryptophan cause sustained increases in the free tryptophan concentration in plasma and 5-hydroxytryptamine synthesis in brain? Biochem. J., 262, 365-368.

17. Miller, L.L., Bly, C.G., Watson, M.L., and Balf, W.F. (1951): The dominant role of the liver in plasma protein synthesis. J. Exp. Med., 94, 431-453.

18. Gibali, M., and Perrier, D. (1975): One compartment models and multicompartment models, in Pharmacokinetics, Marcel Dekker, New York, pp. 1-4, 45-96.

19. Yamaoka, K., Tanigawa, Y., Nakagawa, T., and Uno, T. (1981): A pharmacokinetic program (MULTI) for microcomputer. J. Pharmacobio.-Dyn., 4, 879-885. 